\title{
The Dark Energy Survey Instrument Design
}

\author{
B. Flaugher, for the Dark Energy Survey Collaboration* \\ Fermi National Accelerator Laboratory ${ }^{\dagger}$, Box 500, Batavia, IL, USA 60510
}

\begin{abstract}
We describe a new project, the Dark Energy Survey (DES), aimed at measuring the dark energy equation of state parameter, $w$, to a statistical precision of $\sim 5 \%$, with four complementary techniques. The survey will use a new 3 sq. deg. mosaic camera (DECam) mounted at the prime focus of the Blanco 4m telescope at the Cerro-Tololo International Observatory (CTIO). DECam includes a large mosaic camera, a five element optical corrector, four filters (g,r,i,z), and the associated infrastructure for operation in the prime focus cage. The focal plane consists of $622 \mathrm{~K} \times 4 \mathrm{~K}$ CCD modules $(0.27 \mathrm{H} / \mathrm{pixel})$ arranged in a hexagon inscribed within the $2.2 \mathrm{deg}$. diameter field of view. We plan to use the 250 micron thick fully-depleted CCDs that have been developed at the Lawrence Berkeley National Laboratory (LBNL). At Fermilab, we will establish a packaging factory to produce four-side buttable modules for the LBNL devices, as well as to test and grade the CCDs. R\&D is underway and delivery of DECam to CTIO is scheduled for 2009.
\end{abstract}

Keywords: Dark Energy, CCD, camera, survey, Blanco, CTIO

\section{INTRODUCTION}

The primary scientific goal of the DES is to measure the dark energy equation of state parameter $w$ using four independent and complementary techniques: galaxy cluster counting, baryon oscillations, weak lensing, and using Type Ia supernovae. To make these measurements, the DES Collaboration proposes to build DECam, a new wide field optical CCD camera for the prime focus of the CTIO Blanco $4 \mathrm{~m}$ telescope, and use it to carry out a deep (24 mag.) four-band survey covering $5000 \mathrm{sq}$. deg. of the southern sky. The area and location of the DES are chosen to overlap with the South Pole Telescope project (SPT). The SPT is a 4000 sq. deg. survey of the Southern Galactic Cap that will use the Sunyaev-Zeldovich effect to identify and measure the masses of $\sim 20,000$ galaxy clusters. The SPT is under construction and is expected to begin collecting data in the fall of 2007. The DES optical filter pass bands have been chosen to match the SDSS g,r,i,z filters that have been demonstrated to provide accurate photometric red shift information. The DES survey area will cover the entire SPT area with the goal of acquiring precise red shift measurements of the galaxy clusters identified by SPT. To minimize the photometric uncertainties, the DES survey area will also connect and overlap with the well calibrated area of the sky known as SDSS stripe-82. The combination of the SPT mass estimates and the redshifts from DES provide the basis for the predicted constraints on w from galaxy clusters. In addition, the large survey volume ( 300 million galaxies) will enable precise and complementary constraints on $\mathrm{w}$ from weak lensing and from measurements of baryon oscillations. By using $\sim 10 \%$ of the DES survey time we expect to find $\sim 1900 \mathrm{SN}$ Ia which can provide another complementary constraint on w. Each of these four techniques can constrain w, with 5-15\% statistical errors. Currently w is only constrained at the $30 \%$ level by a combination of different experiments. The DES constraints and systematic uncertainties are described in the DES whitepapers ${ }^{1}$ and supplements that were submitted to the Dark Energy Task Force (DETF) in June 2005. Work is in progress to determine the constraints on w from the combination of the 4 DES methods. The report of the DETF is expected to be released to the Astronomy and Astrophysics Advisory Committee (AAAC) May, 2006. In the language of the DETF findings (presented at the Feb. AAAC meeting) the DES falls into the category of a stage III project: near term and modest cost compared to the stage IV projects such as LSST and SNAP.

\footnotetext{
* Fermilab, University of Illinois at Urbana-Champaign, University of Chicago, Lawrence Berkeley National Lab, NOAO/CTIO, Consejo Superior de Investigaciones Cientificas; Institut d'Estudis Espacials de Catalunya, Institut de Fisica d'Altes Energies, CIEMAT, Madrid; University College London, University of Cambridge, University of Edinburgh, University of Portsmouth, University of Michigan.

${ }^{\dagger}$ Operated by Universities Research Association Inc. under Contract No. DE-AC02-76CH03000 with the United States Department of Energy.
} 
The DES was proposed in response to the National Optical Astronomy Observatory (NOAO) Announcement of Opportunity (AO) in December 2003 that requested proposals for the development of a major new community instrument for the Blanco in partnership with NOAO. The AO offered the instrument collaboration up to $30 \%$ of the observing time (525 nights) over a five-year period and encouraged the construction of an instrument that could exploit the wide field capability of the prime focus. In August 2004, the DES proposal was reviewed by the Blanco Instrumentation Review Panel (BIRP) and subsequently accepted by NOAO. The DES project director and the director of CTIO have drafted an MOU which outlines the terms of the partnership, particularly the allocation of $30 \%$ of the Blanco telescope time over 5 years in exchange for DECam and the DES data analysis infrastructure. Funding for DECam would be provided (if approved) primarily by DOE through Fermilab, with additional major contributions from the international partners (the UK Consortium and the Spanish Consortium) and from the collaborating US Universities. Funding for the development of the data management system and archive is being sought from NSF in combination with startup funds from U. Illinois at Urbana-Champaign (UIUC) and the National Center for Supercomputing Applications (NCSA).

\section{DECAM}

We have developed a design for DECam that requires only modest development of proven technologies with the goal of minimizing the construction time and costs while maximizing the scientific potential. Table 1 contains a summary of the DES survey parameters and the expected performance of the CTIO site and DECam. Figure 2 shows a cross section of DECam and a picture of the Blanco telescope. DECam will replace the entire prime focus cage of the Blanco and attach to the existing spider support fins. The major components of DECam are a 520 megapixel optical CCD camera with vacuum and cryogenic controls, a compact low noise CCD readout system housed in actively cooled crates, a combination shutter-filter system to house the shutter, the four DES filters $(\mathrm{g}, \mathrm{r}, \mathrm{i}, \mathrm{z})$ plus slots for two additional filters that could be provided by the observer community, and a wide-field optical corrector $(2.2 \mathrm{deg}$. field of view $)$. The CCD vessel and corrector are supported as a single unit by a $6 \times 6$ hexapod that will provide lateral adjustability as well as focus control. Note that the prime focus cage of the Blanco can flip such that the F/8 mirror that is mounted to the back of the cage, behind the CCD vessel, points towards the primary mirror for Cassegrain observations. DES will maintain this capability by attaching the existing F/8 mirror to the back of the new DES prime focus cage.

Table 1 Expected performance of DECAM, Blanco, and CTIO site

\begin{tabular}{|c|c|}
\hline Blanco Effective Aperture/ f number @ prime focus & $4 \mathrm{~m} / 2.7$ \\
\hline Blanco Primary Mirror - $80 \%$ encircled energy & $0.25 \operatorname{arcsec}$ \\
\hline Optical Corrector Field of View & $2.2 \mathrm{deg}$. \\
\hline Corrector Wavelength Sensitivity & $<350-1000 \mathrm{~nm}$ \\
\hline Filters & SDSS g, r, i, z (400-1000 nm) \\
\hline Effective Area of CCD Focal Plane & 3.0 sq. deg. \\
\hline Image CCD pixel format/ total \# pixels & $2 \mathrm{~K} X 4 \mathrm{~K} / 520 \mathrm{Mpix}$ \\
\hline Guide, Focus \& Alignment Sensor CCD pixel format & $2 \mathrm{~K} \mathrm{X} 2 \mathrm{~K}$ \\
\hline Pixel Size & $0.27 \operatorname{arcsec} / 15 \mu \mathrm{m}$ \\
\hline Readout Speed/Noise requirement & $250 \mathrm{kpix} / \mathrm{sec} / 10 \mathrm{e}$ \\
\hline $\begin{array}{l}\text { Survey Area } \\
\text { SPT overlap } \\
\\
\text { SDSS stripe } 82 \\
\text { Connection region }\end{array}$ & $\begin{array}{l}5,000 \text { sq. deg. total } \\
\text { RA }-60 \text { to } 105, \text { DEC }-30 \text { to }-65 \\
\text { RA }-75 \text { to }-60, \text { DEC }-45 \text { to }-65 \\
\text { RA }-50 \text { to } 50, \text { Dec }-1 \text { to } 1 \\
\text { RA } 20 \text { to } 50, \text { Dec }-30 \text { to }-1\end{array}$ \\
\hline Survey Time/Duration & $525 / 5$ (nights/years) \\
\hline Median Site Seeing Sept. - Feb. & $0.65 \operatorname{arcsec}$ \\
\hline Median Delivered Seeing with Mosaic II on the Blanco & $0.9-1.0 \operatorname{arcsec}(\mathrm{V}$ band $)$ \\
\hline Limiting Magnitude: $10 \sigma$ in 1.5 " aperture assuming 0.9 " seeing, AB system & $\mathrm{g}=24.6, \mathrm{r}=24.1, \mathrm{i}=24.3, \mathrm{z}=23.9$ \\
\hline Limiting Magnitude: $5 \sigma$ for point sources assuming 0.9 " seeing, AB system & $\mathrm{g}=26.1, \mathrm{r}=25.6, \mathrm{i}=25.8, \mathrm{z}=25.4$ \\
\hline
\end{tabular}


The DES survey strategy is based on taking multiple short exposures $(100 \mathrm{sec})$ and adding them together to reach the required depth in each filter pass-band. This strategy minimizes the systematic uncertainties resulting from effects such as atmospheric variations. In first two years we plan to tile the entire survey area multiple times to enable early scientific results. To maximize the total time available for exposures, we plan to read out the image CCDs while the telescope is slewing to a new position. Current measurements indicate that the time it takes for a typical DES slew of 2 deg. is $\sim 35 \mathrm{sec}$. Upgrades planned by CTIO for the telescope control system ${ }^{2}$ will likely reduce this to $\sim 20 \mathrm{sec}$. A CCD readout rate of $250 \mathrm{kpix} / \mathrm{sec}$ will take $17 \mathrm{sec}$ and fit within the telescope slew time.

We plan to read out the alignment and focus CCDs with the image CCDs to provide the possibility for image by image corrections. Currently at the Blanco, observations are interrupted 2-3 times/night to correct the telescope focus and the corrector-primary mirror alignment is checked and adjusted every few months. Experience with DECam on the Blanco will determine the frequency at which corrections are needed to maintain the best image quality. The guide CCDs need to provide signals to the telescope controls system at a rate of $\sim 1 \mathrm{~Hz}$. To achieve this rate, exposures of $\sim 0.5 \mathrm{sec}$ are envisioned and only a small area centered on the guide star will be read out.

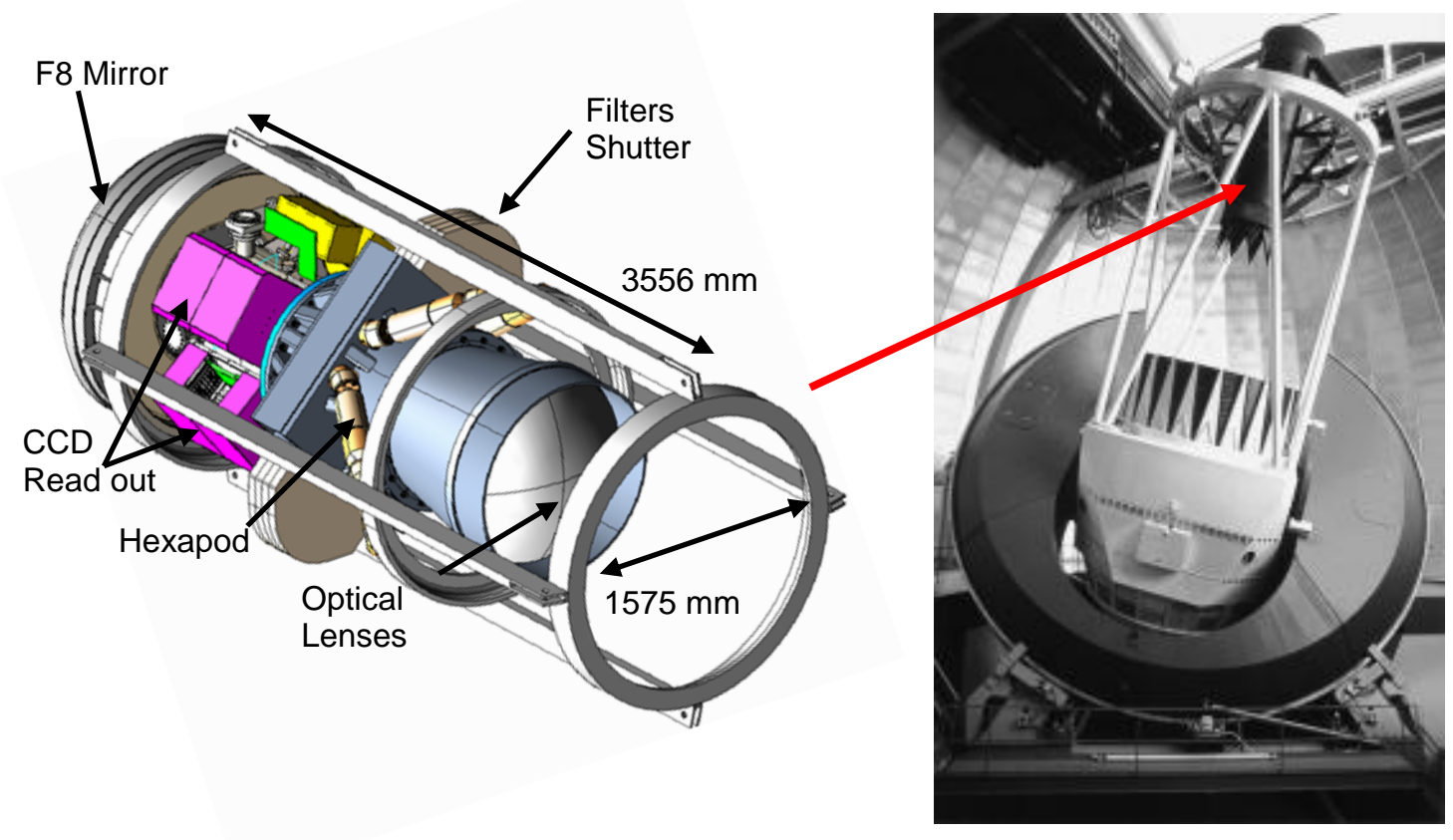

Figure 1 Isometric view of the DECam, the DES prime focus instrument with a picture of the Blanco telescope.

\subsection{DES Focal plane}

To efficiently obtain $z$-band images for high-redshift ( $\mathrm{z} \sim 1.3$ ) galaxies, we have selected the fully depleted, highresistivity, 250 micron thick $\mathrm{CCDs}^{3}$ that have been designed and developed at the LBNL. The thickness of the LBNL design has two important implications for DES: fringing is eliminated, and the QE of these devices is $>50 \%$ in the $z$ band, a factor of 5-10 higher than traditional thinned astronomical devices. Figure 2 shows the QE of the LBNL CCDs compared to the CCDs currently in the Mosaic II camera on the Blanco. Several of the LBNL 2k x 4k CCDs of this design have been successfully used on telescopes, including the Mayall $4 \mathrm{~m}$ at Kitt Peak and the Shane $3 \mathrm{~m}$ at Lick. Measurements at LBNL have shown that the point spread function (PSF) obtained by these devices is comparable to the more traditional 20-30 micron thick CCDs such as those available from E2V. The read noise of the CCDs is a function of speed. At a rate of $250 \mathrm{kpix} / \mathrm{sec}$ LBNL has measured a read noise of $7 \mathrm{e}$, which is below the DES requirement of $<10 \mathrm{e}$. 


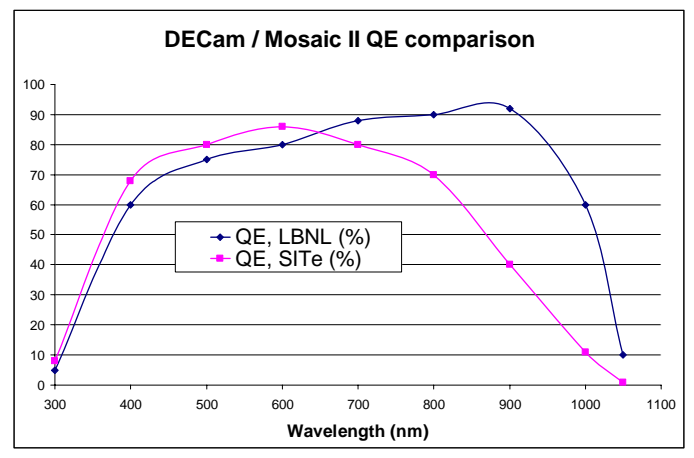

Figure 2 Quantum Efficiency of the LBNL CCDs and the CCDs in Mosaic II

LBNL developed this CCD design in the MicroSystems Lab processing facility at LBNL on 4" wafers. For DES we have chosen to follow the CCD acquisition model that has been developed by LBNL for the SNAP devices. In this business model, the wafer vendor performs the first 8 steps in the CCD processing on 650 micron thick 6" diameter wafers. The wafers are then delivered to LBNL for thinning to 250 microns and the final three process steps. For DES a new layout, shown in Figure 3, containing four $2 \mathrm{kx} 4 \mathrm{k}$ devices on a single 6" wafer was required to keep the processing costs to a minimum (previous wafer layouts had only two $2 \mathrm{kx} 4 \mathrm{k}$ devices per wafer). The DES wafer layout also incorporates a $2 \mathrm{kx} 2 \mathrm{k}$ device and eight $0.5 \times 1 \mathrm{k}$ devices. The wafer layout was completed in the spring of 2005 and the first devices from the DES wafers were delivered to LBNL in the summer of 2005 . The $2 \mathrm{kx} 4 \mathrm{k}$ devices on the fully processed wafers are tested on a cold probe station at LBNL, then shipped out for dicing and delivery to Fermilab. The first fully processed 250 micron thick devices arrived at Fermilab in November 2005. These engineering grade devices are being used for development of the packaging and testing procedures. Reference 4 describes the status and preliminary results of the CCD characterization. The first science grade DES devices are expected in Oct. 2006. The preliminary estimate of the yield for science grade devices is $25 \%$ based on an LBNL study of devices procured with this business model for SNAP.

The DES focal plane, also shown in Figure 3, will consist of sixty-two $2 \mathrm{~K}$ x $4 \mathrm{~K}$ CCDs $(0.27 \% /$ pixel $)$ arranged in a hexagon covering an imaging area of 3 sq. degrees. Smaller format CCDs for guiding $(G)$, focusing $(F)$ and alignment (WF) are located at the edges of the focal plane.
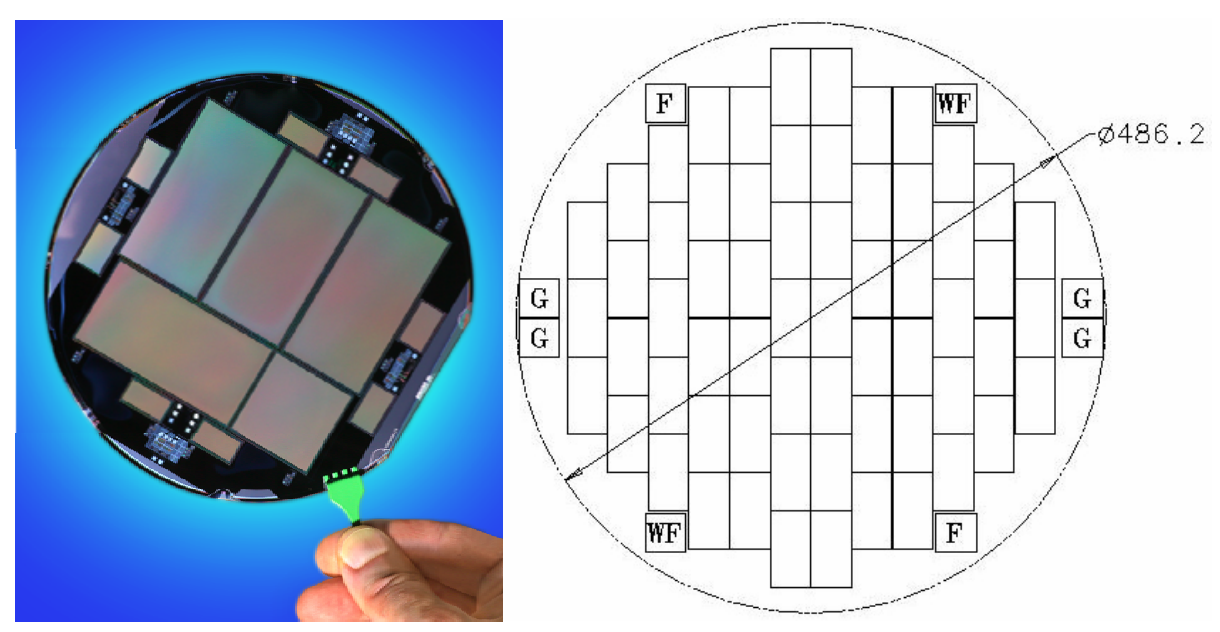

Figure 3 A wafer of DES CCDs showing the $2 \mathrm{kx} 4 \mathrm{k}, 2 \mathrm{kx} 2 \mathrm{k}$ and small test CCDs. The DES Focal plane is shown on the right. 
The DES CCDs will be packaged and tested at Fermilab, capitalizing on the experience and infrastructure associated with construction of silicon strip detectors for the Fermilab Tevatron program. The four-side buttable package for the $2 \mathrm{k}$ $\mathrm{x} 4 \mathrm{k}$ devices builds on techniques developed by LBNL and Lick Observatory. The DES science requires a uniform PSF. This translates into a requirement that each CCD module have a flatness (peak-to-peak) of $<10$ microns. Careful matching of the thermal expansion coefficients is required to achieve this level of flatness when the CCD modules are cooled to an operating temperature between -100 and -150 deg.C. In the prototype focal plane package, called the pedestal package, the front side of the CCD is glued and wire-bonded to an Aluminum Nitride (AIN) circuit board, then to an AlN spacer and an Invar foot. A 37 pin connector is located in the center of the device and is accessed through a hole in the foot. Due to the large number of devices that will need to be packaged and tested, an emphasis on ease of assembly and handling will be incorporated into the design. Reference 5 describes the status of the DES CCD packaging.

\subsection{CCD Readout}

CCD testing and characterization is currently being performed at Fermilab using the Monsoon readout system ${ }^{6}$ developed by NOAO. The characterization of the CCDs at LBNL used a Leach controller and we have also setup a similar system at Fermilab to provide cross checks and comparisons of performance. In March 2006 we achieved 6.5e noise operations with the Monsoon system and a DES CCD at a readout speed of $160 \mathrm{kpix} / \mathrm{sec}$. Although the Monsoon architecture was designed to be compact and low power, it is still too large to accommodate the number of channels required by DES at the prime focus and we are investigating a number of options. The furthest developed is a compressed design based on Monsoon, but customized to meet the specific needs of the DES.

Each DES CCD has two video readout channels (U/L) and requires connection to 15 clock inputs, and 7 bias voltages. In addition, each CCD has a 4-wire temperature sensor on the AlN circuit board that will be used to track the CCD temperature during operation. The NOAO Monsoon system consists of 3 boards: a Master Control Board (MCB), a Clock and Bias Board (CBB) and an 8-channel video card (ACQ). The MCB is used to communicate with host computer and control the crate. The communication link is via Systran FibreXtreme SL100/SL240 Series cards. The CBB produces 32 clocks, 36 bias voltages and 8 fast bias voltages. The 8 -channel card produces 32 Bias voltages and has 8 analog channels. To fit in the prime focus cage, we have assumed that the CCD performance will not be degraded if 1) the clock signals on both sides of the CCD are tied together and 2) these signals are common to groups of 3 CCDs. This will allow each CBB to provide the clock signals for 9 CCDs. We are also compressing the 8 channel ACQ board design to make room for a total of 12 analog channels. This 12 channel board will also contain the circuitry to handle the temperature sensors on each device. With these modifications the readout for the DES focal plane will fit into three 10 slot dual backplane crates. To provide guide signals to the telescope, the guide CCDs will be operated independently of the image CCDs by locating their readout on a separate backplane. Each 10 slot crate is split into a 4-slot and a 6-slot backplane. A 4-slot backplane can readout up to 9 CCDs and will contain one MCB, one CBB and two ACQ. Each 6slot backplane can read out up to 18 CCDs and will contain one MCB, two CBB and three ACQ. The guide CCDs will occupy one of the 4-slot backplanes. The remaining backplanes can accommodate the readout for up to 72 CCDs. Further options for customization for DES include removing the unused bias circuitry from the CBB and replacing the Systran communications link with an S-LINK ${ }^{7}$ system.

The CCD readout crates will be located in thermally controlled housings. These housings will maintain the CCD electronics at a constant temperature to ensure constant gain performance. The outside of the housing will follow the ambient night temperature to avoid creation of thermal plumes in the optical path. A liquid coolant, likely a water alcohol mixture, will be transported to these housings through insulated lines to remove the heat from the electronics.

\subsection{CCD Camera}

The camera vessel contains the last element of the optical corrector (C5, see below) and the CCD focal plane array. The current design uses a Liquid Nitrogen (LN2) dewar that is connected to the focal plane support plate by copper straps. Each strap will have a temperature monitor and a heater that can tune the temperature of the focal plane support plate. The CCD operating temperature will be between $-150 \mathrm{deg}$. $\mathrm{C}$ and $-100 \mathrm{deg}$. $\mathrm{C}$ and will be determined by study of the quantum efficiency as a function of temperature. Finite element models indicate that with 12 straps the focal plane support plate temperature can be uniform to $<2 \mathrm{deg}$. C across the $525 \mathrm{~mm}$ diameter focal plane. The C5 lens is athermally constrained within a stainless steel cell using radial High Density Polyethylene (HDPE) spacers that are sized to compensate for the CTE difference between the lens and the cell. The C5 cell mounts to the front flange of the camera vessel with an o-ring seal between the lens and the flange. The CCD array is attached to the vessel using bipod style 
mounts. The bipods mounts thermally isolate the array from the rest of the vessel and locate the array with respect to C5. The CCD vessel has a $61 \mathrm{~cm}$ diameter and is approximately $84 \mathrm{~cm}$ inches in the axial direction. Figure 4 shows the CCD vessel along with the CCD readout crates that are mounted to the outside of the vessel. The cooling straps, bipods and the feed-thru board for the CCD readout signals is shown on the left side of the figure. A full sized prototype camera vessel is currently under construction. It will provide the test bed for multi CCD readout in an environment very close to what is imagined for the final CCD vessel design.

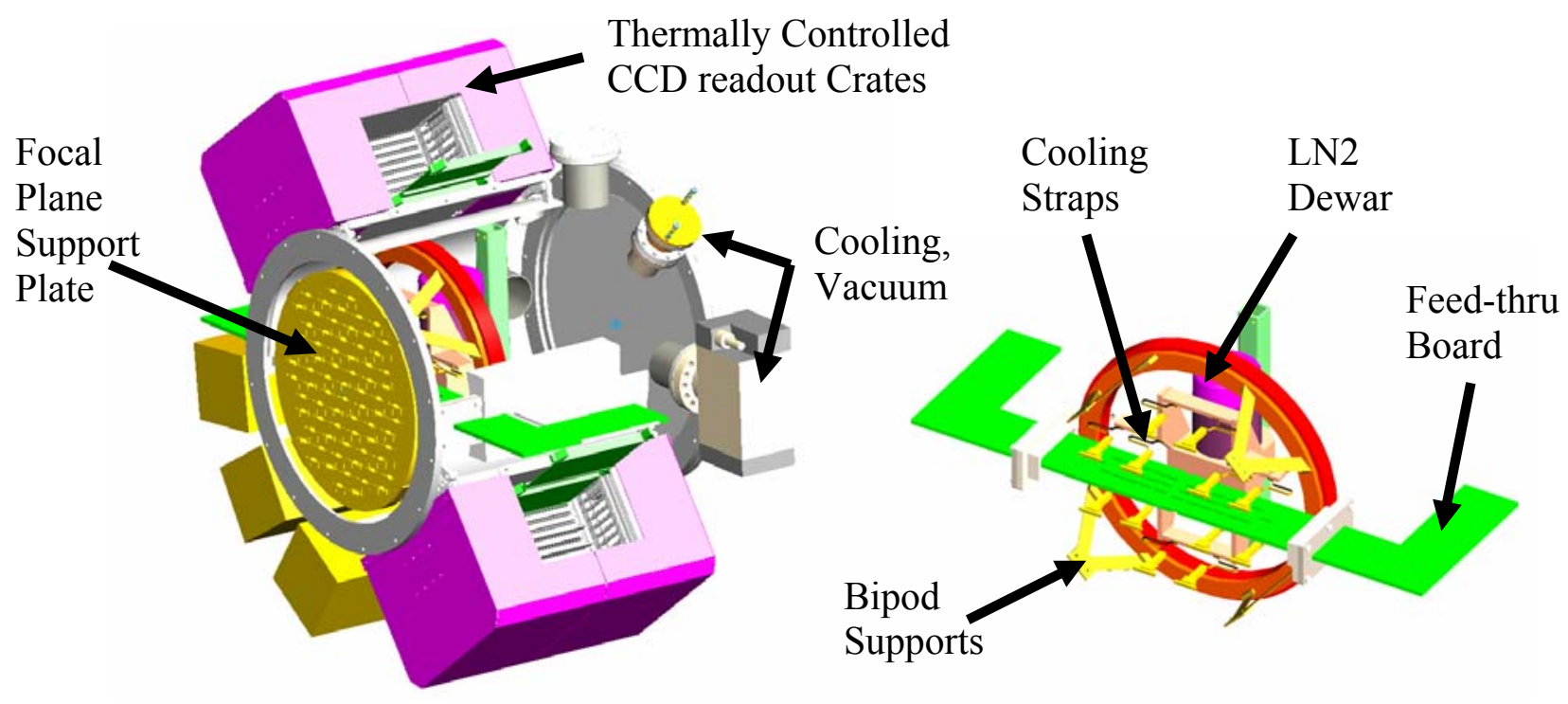

Figure 4 Cutaway view of the DES CCD vessel and a view of the internal features.

\subsection{Optical Corrector}

The optical corrector design ${ }^{8}$ consists of five fused silica lenses that produce an unvignetted $2.2^{\circ}$ diameter image area. The largest element (C1) is $930 \mathrm{~mm}$ diameter. The last element (C5) is the window on the CCD vacuum vessel. The spacing between elements 3 and 4 allows room for the $\sim 570 \mathrm{~mm}$ diameter filters to slide in and out of the optical path. To maximize the stiffness of the corrector barrel the shutter will be combined with the filter changer. Studies indicate that an Atmospheric Dispersion Corrector (ADC) would not significantly improve the optical performance for DES and thus the DECam optical corrector does not include one. Details of the DES optical design are presented in Reference 8. A schematic of the corrector elements is shown in Figure 5.

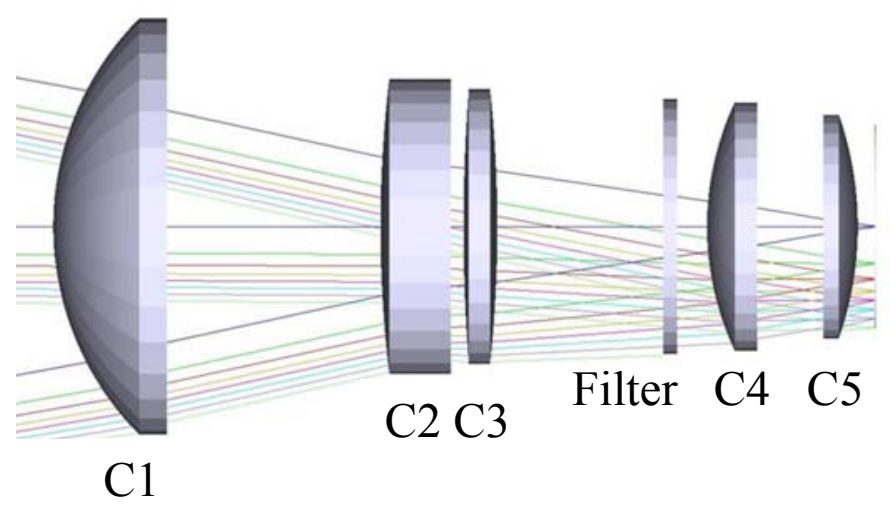


Figure 5 The DECAM optical corrector

The lenses will be mounted into cells and then into the corrector barrel. Detailed design of the cells and the barrel is in progress. A conceptual design of the corrector barrel is shown in Figure 6. The corrector barrel will consist of two large pieces that bolt together. A conical section supports $\mathrm{C} 1$. A barrel section supports $\mathrm{C} 2, \mathrm{C} 3$, and $\mathrm{C} 4$ as well as the combined filter-shutter system. In earlier designs the shutter was located at the end of the barrel section, just in front of $\mathrm{C} 5$, at the connection between the corrector and the CCD vessel. The new location, just in front of $\mathrm{C} 4$ allows significant improvement to the strength of connection between the corrector and the CCD vessel.

The material chosen for the corrector barrel is steel and the weights of the various DES components are shown in Table 2. A preliminary Finite Element Model (FEM) of the barrel and CCD vessel has been used to estimate the deflections of each of the lenses under gravity loading. Reinforcing tubes have been added around the filter-shutter system with the goal of keeping the deflections of $\mathrm{C} 1$ and the focal plane to $<25$ microns. The results of the FEM have been fed into a sensitivity analysis of the optics to generate an estimate of the resulting changes to the image quality and the initial indications are that the design is sufficiently stiff. Future work will include a more detailed FEM and will investigate making the barrel and CCD vessel stiffer and lighter.

Table 2 Weight of the DES components

\begin{tabular}{|l|l|}
\hline Component & Weight (kgs) \\
\hline Barrel & 820 \\
\hline CCD vessel inc. C5 & 590 \\
\hline C1,C2,C3,C4 + cells & 475 \\
\hline Filter+Shutter Cartridge & 390 \\
\hline & \\
\hline Total & $\mathbf{2 2 7 5}$ \\
\hline
\end{tabular}
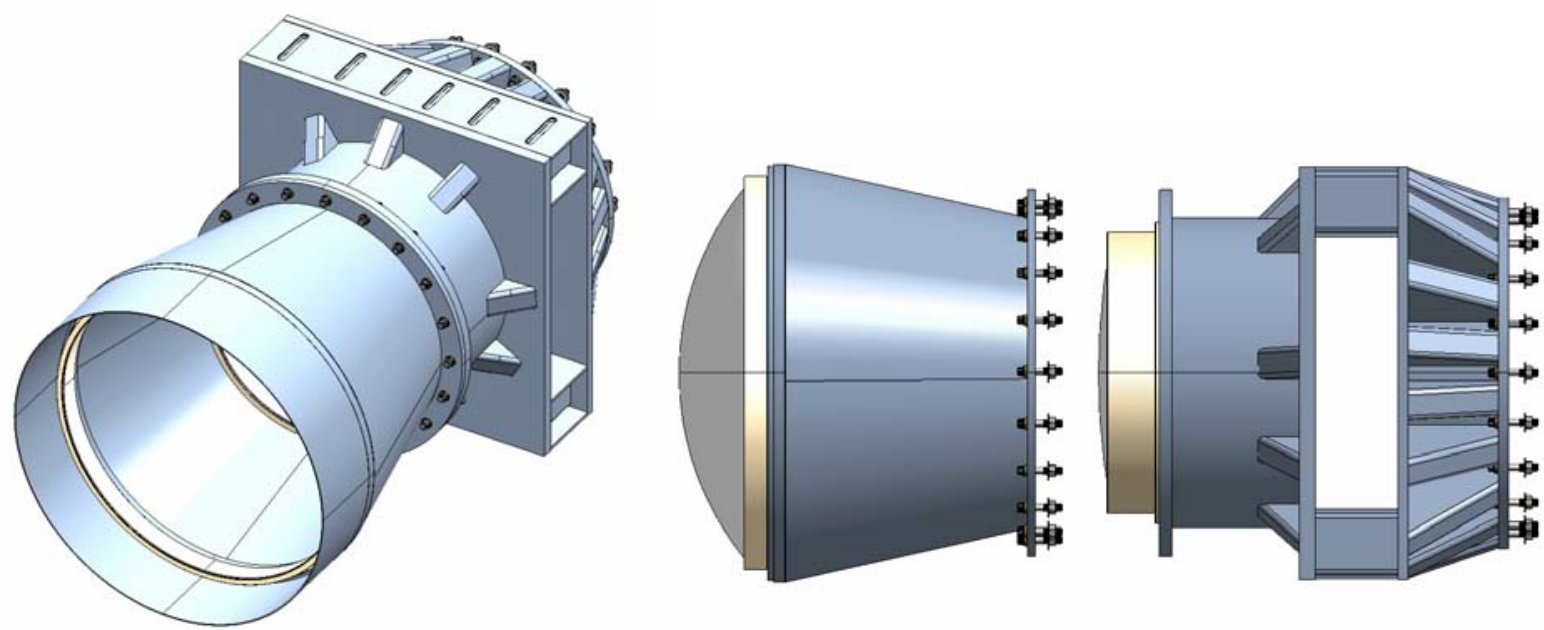

Figure 6 Conceptual Design of the DES Corrector Barrel 
The leading candidate for the DECam shutter is a design developed by the Hoher List Observatory, at Bonn University. The shutter has sliding blades (a "flying slit") for fast exposures and to obtain equal exposure times in both directions of travel. The DES filters will be $\sim 570 \mathrm{~mm}$ diameter and $20-30 \mathrm{~mm}$ thick. The main challenge in filter fabrication is expected to be uniformity over this large area. Other projects such as PanSTARRS, LSST, the WIYN ODI and the Discovery Channel Telescope have similar requirements. DES has developed a set of detailed filter specifications that has been sent out for budgetary quotes.

The filter cartridge will house the four DES filters and up to two guest filters. In this design, there are three cassettes in the cartridge. Each cassette houses two filters with one filter at each end of the cassette. The cartridge and the corrector barrel are purged with dry nitrogen to maintain a constant humidity environment. The cartridge system places the filters at different positions in the optical path. Initial feedback from the filter vendors indicate that the filters may be as thick as $30 \mathrm{~mm}$ and with the addition of the mechanical housings this will produce $\sim 50 \mathrm{~mm}$ offsets between the filters in different cassettes. The optical beam is roughly a constant diameter in this region and we expect that all the filters will be the same size. An advantage of the cartridge system is that it will minimize the risk and time associated with changing between the DES filters and those needed by the community users of the camera.

\subsection{Alignment and Focus}

The current conceptual design for the support and position adjustment of the camera/barrel assembly in the prime focus cage incorporates a 6-6 hexapod. As shown in Figure 1, the base of the hexapod would be mounted to a ring built into the prime focus cage. The corrector would be attached to the top of the hexapod using the front plate of the filter/shutter assembly. Note that the CCD vessel is aligned and bolted to the corrector and the combination camera/barrel system moves as a single unit. A hexapod system would provide both the focus adjustments and has the capability to provide additional degrees of freedom, if it were deemed necessary to keep the corrector aligned to the primary mirror. Serial/stacked adjustable support systems are also being evaluated as an alternative to the hexapod.

The focus and alignment CCDs will be mounted either above and below the nominal surface of the focal plane, or at an angle with respect to the focal plane. Comparison of the out-of-focus images above and below the focal plane will indicate the direction of motion needed to bring the image back into focus. The alignment CCDs and a variant of the "donut" method now under development at CTIO for the SOAR telescope will be used to monitor the alignment of the corrector to the primary mirror. The focus and alignment CCDs will be read out with the image CCDs providing image by image information on the telescope focus and alignment.

In Oct. 2005, digital micrometers were installed on the primary mirror. These allow constant monitoring of the mirror position and movements. DES also plans to install a $\mathrm{BCAM}^{9}$ system which will directly measure the relative positions of the mirror and the corrector without needing to operate the CCDs. Information from one or both of these systems will be used to determine if the corrector needs to be moved laterally in the cage to be better aligned with the primary mirror.

\subsection{Survey Image System Process Integration (SISPI)}

The mountain top software in DES is called Survey Image System Process Integration (SISPI). This is the set of processes that will control the image acquisition and deliver the images to the data management system for processing. Figure 7 shows the relation between the various processes that directly control CCD digitization as well as the data and control flow between them. The processes that CTIO uses for telescope and dome control have been omitted for simplicity. DES will have about a dozen computers on the mountain devoted to different aspects of the SISPI. The DES computing environment will be similar to other existing systems such as SDSS although our cadence (exposure sequencing) and data rates will be different. The diagram is hierarchical to encapsulate interfaces and local data flow. At the right, one bubble is shown expanded.

The software integration can be divided into two kinds of work: subsystem specific software needed for device functionality and a common infrastructure to enable inter-system communication and data flow. To avoid chaos, the design work has proceeded in stages. Last fall, the collaboration wrote a Functional Requirements Document (FRD) that described what each part of the system should do. Given that document, we are now in the midst of writing an Interface Control Document (ICD) that defines more precisely the commands and data that must flow between processes. It 
should be complete this summer and will contain enough detail that implementation of each bubble can then be left to the individual groups that are responsible.

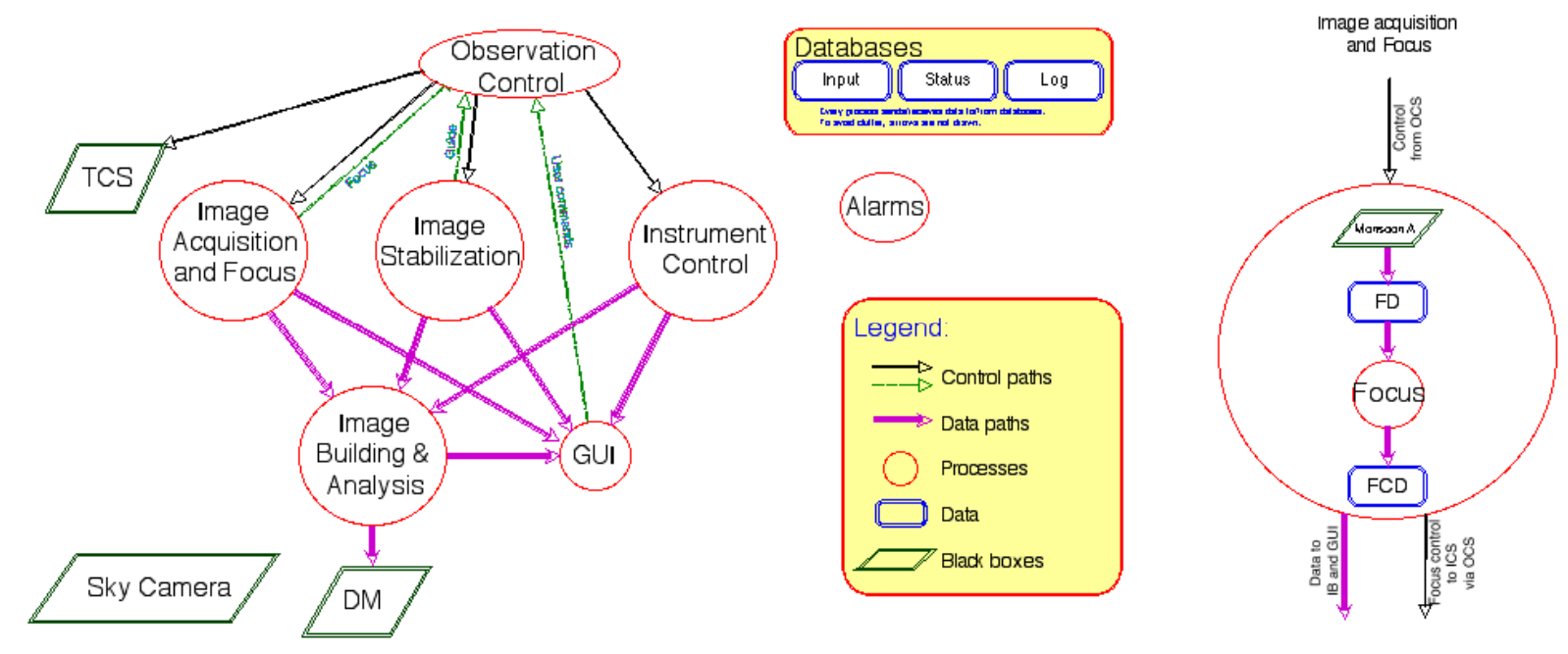

Figure 7 A schematic diagram of the DES SISPI system.

\section{DES PROJECT STATUS AND SCHEDULE}

A detailed schedule for the DES project was prepared and reviewed in June 2004 as part of both the Fermilab and NOAO project review process. The procurement of the CCDs and the optical elements were identified as the critical path items for the project. Since those reviews, we have focused our resources on addressing these topics. We are on a schedule for delivery of DECam to CTIO 2009, assuming that funding from the several national agencies can be solidified over the next year.

The optics are on the critical path primarily because of the lengthy (2-2.5yr) estimated procurement. We held a Preliminary Review of the Optical design in Feb. 2006 to obtain early feedback from external reviewers. Their response to the DES design was positive and they urged us to move forward with the rest of the mechanical design. We plan to have a complete review of the DES project in July 2006 with the goal of being ready to place the order for the glass blanks in August, or as soon as the funding is secured.

The CCDs are on the critical path due to the estimated delivery rate (5 wafers per month plus a 3 month startup) combined with the uncertainty in the CCD yield. For example, assuming the yield is $25 \%$, production of 70 good devices would require $\sim 18$ months of processing, packaging and testing. By Dec. 2006 we should have more information on the CCD yield. This will feed directly into the project cost and schedule.

\section{CONCLUSIONS}

The Dark Energy Survey Collaboration plans to build a new powerful CCD camera, install it on the Blanco 4m telescope at CTIO and use it to measure the dark energy equation of state parameter, w, using 4 complementary techniques. The 3 sq. deg camera will be 7 times larger and 7 times faster than the prime focus camera currently on the Blanco and will use CCDs that are $\sim 10$ times more sensitive in the z-band that traditional CCDs. The DECam design builds on existing 
technologies to minimize development time. If funding can be secured in the next year, DECam could be on the telescope in 2009.

\section{REFERENCES}

1 The Dark Energy Survey whitepaper; submitted to the Dark Energy Task Force June 2005, astro-ph/0510346. Additional whitepapers on Theoretical \& Computational Challenges, submitted to the Dark Energy Task Force Sept. 2005, astro-ph/0510194,5; also available on the web: https://www.darkenergysurvey.org/the-project/survey_documents/DES-DETF/.

2 See "The CTIO V. M. Blanco 4-m Telescope and the Dark Energy Survey" T. Abbott et al in these proceedings.

3 Holland, S. et al. IEEE Trans. Elec. Dev., 50, 225 (2003).

4 "Testing and Characterization of CCDs for the Dark Energy Survey" J. Estrada et al, in these proceedings

5 "The Status of 0.250mm-Thick CCD Packaging for the Dark Energy Survey Camera Array" G. Derylo et al in these proceedings

6 Information on the Monsoon CCD readout system is available at: http://www.noao.edu/ets/monsoon/

7 For Information on S-LINK see: http://hsi.web.cern.ch/HSI/s\%2Dlink/

8 “The Dark Energy Survey Optical Corrector Design”, S. Kent, et al. in these proceedings.

9 For information on the BCaMs see http://alignment.hep.brandeis.edu/Devices/BCAM/. 